\title{
FREE PROBABILITY AND QUANTUM ELECTRODYNAMICS
}

\author{
L. ACCARDI \\ Centro V. Volterra, Università di Roma "Tor Vergata", \\ Via Columbia 2, 00133 Roma, Italia \\ (e-mail: accardi@volterra.mat.uniroma2.it) \\ and \\ Y. G. LU \\ Dipartimento di Matematica, Università di Bari, \\ Via Orabona 4, 70125 Bari, Italia \\ (e-mail: lu@dm.uniba.it)
}

(Received October 13, 2003 - Revised January 30, 2004)

\begin{abstract}
The stochastic limit of a free particle coupled to the quantum electromagnetic field without dipole approximation leads to many new features such as: interacting Fock space, Hilbert module commutation relations, disappearance of the crossing diagrams, etc. In the present paper we begin to study how the situation is modified if a free particle is replaced by a particle in a potential which is the Fourier transform of a bounded measure.

We prove that the stochastic limit procedure converges and that the overall picture is similar to the free case with the important difference that the structure of the limit Hilbert module is strongly dependent on the wave operator of the particle.
\end{abstract}

Keywords: stochastic limit, quantum electrodynamics, scattering operator, Hilbert module, interacting Fock space.

MSC: $60 \mathrm{H} 40$

\section{Introduction}

The stochastic limit of quantum electrodynamics has been studied systematically in a series of papers dealing with a multiplicity of physical models. The main result in those papers can be stated as follows: if we drop the dipole approximation on the quantum EM field, in order to understand asymptotically its evolution, one needs a quantum stochastic process, which is the solution of a white noise Hamiltonian equation (equivalent to a quantum stochastic differential equation) driven by a quantum white noise living on some Hilbert module. Moreover, the quantum noise is

i) bosonic, if we introduce the (quasi) dipole approximation;

ii) an entangled extension of the free white noise, if we drop the dipole approximation.

In previous investigations [1-3] we have considered a free particle, i.e. a particle driven by the kinetic energy minimally coupled to the EM field. Now we are going 
to investigate the same problem in the case of a particle driven by kinetic and potential energy.

Let be given a physical system (e.g. an atom), described by a Hilbert space $\mathbf{L}^{2}\left(\mathbb{R}^{d}\right)$ with $d \geq 3$ and a Hamiltonian operator

$$
H_{S}:=p^{2}+V(q),
$$

where $p$ is the momentum operator, $q$ the position operator and $V$ is a potential function in the Schwartz class. The particle is assumed not to be spatially confined and therefore its kinetic energy spectrum is continuous. Suppose that this system is coupled to the quantum EM field (whose Hamiltonian will be denoted by $H_{R}$ ) via the interaction Hamiltonian

$$
H_{I}:=\lambda p \sum_{k} e^{i k \cdot q} a_{k} \frac{1}{\sqrt{|k|}}-\text { h.c. }
$$

This coupling arises from the minimal coupling by neglecting polarization and the second-order term in $\lambda$ (cf. [3]). In (1.2) $p$ denotes a fixed component of momentum on the orthogonal space to $k$.

In the interaction picture, the evolution of this system is determined by the operator

$$
U_{t}^{(\lambda)}:=e^{i t\left(H_{S} \otimes 1+1 \otimes H_{R}\right)} e^{-i t\left(H_{S} \otimes 1+1 \otimes H_{R}+\lambda H_{I}\right)},
$$

where the coefficient $\lambda$ is called a coupling constant. $\left\{U_{t}^{(\lambda)}\right\}_{t \geq 0}$ defined in (1.3) satisfies the ordinary differential equation

$$
\frac{d}{d t} U_{t}^{(\lambda)}=-i \lambda H_{I}(t) U_{t}^{(\lambda)}
$$

where $H_{I}(t)$ is the evolved interaction Hamiltonian defined by

$$
H_{I}(t):=e^{i t\left(H_{S} \otimes 1+1 \otimes H_{R}\right)} H_{I} e^{-i t\left(H_{S} \otimes 1+1 \otimes H_{R}\right)} .
$$

By a standard procedure [3] (introducing a cut-off and changing the sum to integration) we can rewrite the evolved interaction Hamiltonian as

$$
H_{I}(t)=i\left(\int_{\mathbb{R}^{d}} e^{i t H_{S}}(-i p) e^{i k \cdot q} e^{-i t H_{S}} \otimes \overline{\left(S_{t} g\right)}(k) a_{k}-\text { h.c. }\right),
$$

where $g$ is a Schwartz function, depending on the cut-off; $\left\{S_{t}\right\}_{t \in \mathbb{R}}$ is a unitary group (1-particle free evolution) on the Hilbert space $L^{2}\left(\mathbb{R}^{d}\right)$ coming from the field Hamiltonian and satisfying the fundamental condition: for any $f, g \in \mathcal{S}\left(\mathbb{R}^{d}\right)$ (the Schwartz space of rapidly decreasing functions)

$$
\int_{\mathbb{R}^{d}}\left|\left\langle f, S_{t} g\right\rangle\right| d t<+\infty
$$

The dipole approximation consists in replacing of the factor $e^{i k \cdot q}$ by 1 (or by the first few terms of its Taylor expansion). The starting point of our investigation is to keep this factor and seek for a new, not so drastic, approximation. 
The main result in this article is that, as $\lambda \rightarrow 0$, the operator $U_{t / \lambda^{2}}^{(\lambda)}$ is approximated by a quantum stochastic process $U(t)$. Moreover

i) the convergence is a quantum analogue of the convergence in law;

ii) the limit process $U(t)$ satisfies a quantum stochastic differential equation;

iii) the quantum stochastic differential equation is driven by a new type of quantum noise on a Hilbert module;

iv) the Hilbert module is related to the $\mathrm{M} \phi$ ller wave operator.

The presence of the M $\phi$ ller wave operator represents the main new feature, with respect to the case of a free particle, studied in [3]. For this reason in the following section we describe the main idea of the emergence of this new feature. In Section 3 we describe the limit Fock module and in Section 4 we prove an explicit formula for the mixed moments of the module creation and annihilation process. Finally, in Section 5 we deduce the explicit form of the module stochastic equation for the limit operator. The proofs of the last three sections have not been included because, although lengthy, they do not introduce substantial new ideas with respect to [3].

\section{Some preliminary remarks}

We begin our investigation from the formal solution of Eq. (1.3),

$$
U_{t / \lambda^{2}}^{(\lambda)}=\sum_{n=0}^{\infty}(-i \lambda)^{n} \int_{0}^{t / \lambda^{2}} d t_{1} \int_{0}^{t_{1}} d t_{2} \cdots \int_{0}^{t_{n-1}} d t_{n} H_{I}\left(t_{1}\right) H_{I}\left(t_{2}\right) \cdots H_{I}\left(t_{n}\right) .
$$

In the following, for notational convenience, we shall exchange the order of the system part and the field part, i.e. we rewrite the evolved interaction Hamiltonian $H_{I}(t)$ as

$$
i\left(\int_{\mathbb{R}^{d}} \overline{\left(S_{t} g\right)}(k) a_{k} \otimes e^{i t H_{S}}(-i p) e^{i k \cdot q} e^{-i t H_{S}}-\text { h.c. }\right) .
$$

Thus, for each $n \geq 0$, one has that

$$
\begin{gathered}
(-i)^{n} H_{I}\left(t_{1}\right) H_{I}\left(t_{2}\right) \cdots H_{I}\left(t_{n}\right)=\sum_{\varepsilon \in\{0,1\}^{n}} \int_{\mathbb{R}^{n d}} a_{k_{1}}^{\varepsilon(1)} \cdots a_{k_{n}}^{\varepsilon(n)}\left(S_{t_{1}} g\right)^{\varepsilon(1)}\left(k_{1}\right) \cdots\left(S_{t_{n}} g\right)^{\varepsilon(n)}\left(k_{n}\right) \\
\otimes e^{i t_{1} H_{S}}\left\{(-i p) e^{i k_{1} \cdot q}\right\}^{\varepsilon(1)} e^{-i t_{1} H_{S}} \cdots e^{i t_{n} H_{S}}\left\{(-i p) e^{i k_{n} \cdot q}\right\}^{\varepsilon(n)} e^{-i t_{n} H_{S}},
\end{gathered}
$$

where for any operator $b$ we use the notation

$$
b^{\varepsilon}:= \begin{cases}b, & \text { if } \varepsilon=0 \\ b^{*}, & \text { if } \varepsilon=1\end{cases}
$$

and for any function $F$,

$$
F^{\varepsilon}:= \begin{cases}F, & \text { if } \varepsilon=1 \\ \bar{F}, & \text { if } \varepsilon=0\end{cases}
$$


For example, if $n=1$, (2.2) becomes

$$
-i H(t)=\int_{\mathbb{R}^{d}} a_{k} \overline{\left(S_{t} g\right)}(k) \otimes e^{i t H_{S}}(-i p) e^{i k \cdot q} e^{-i t H_{S}}-\text { h.c. }
$$

The collective two-point function is

$$
\begin{aligned}
\lambda^{2} \int_{0}^{t / \lambda^{2}} d t_{1} \int_{0}^{s / \lambda^{2}} d t_{2}\left\langle 0\left|\int_{\mathbb{R}^{2 d}} d k_{1} d k_{2} a_{k_{1}} \overline{\left(S_{t_{1}} f\right)}\left(k_{1}\right) a_{k_{2}}^{+}\left(S_{t_{2}} g\right)\left(k_{2}\right)\right| 0\right\rangle \times \\
=\lambda^{2} \int_{0}^{t / \lambda^{2}} d t_{1} \int_{0}^{s / \lambda^{2}} d t_{2} \int_{\mathbb{R}^{d}} \overline{(-i p) e^{i k_{1} \cdot q} e^{-i t_{1} H_{S}} \cdot e^{i t_{2} H_{S}} e^{-i k_{2} \cdot q}(i p) e^{-i t_{2} H_{S}}(k)\left(S_{t_{2}} g\right)(k) d k \times} \\
e^{i t_{1} H_{S}}(-i p) e^{i k \cdot q} e^{-i t_{1} H_{S}} \cdot e^{i t_{2} H_{S}} e^{-i k \cdot q}(i p) e^{-i t_{2} H_{S}},
\end{aligned}
$$

where $f, g \in \mathcal{S}\left(\mathbb{R}^{d}\right)$. From the CCR, one knows that (2.6) is equal to (2.7).

In the product of operators in (2.7), it is convenient to move the operator $e^{i k \cdot q}$ until it is erased by meeting the operator $e^{-i k \cdot q}$. This requires the following lemma.

LEMMA 2.1. For any $t \geq 0$,

$$
e^{i k \cdot q} e^{i t H_{S}}=e^{i t\left(H_{S}+p \cdot k\right)} e^{i k \cdot q} e^{i t|k|^{2 / 2}}
$$

Proof: One has

$$
e^{i k \cdot q} e^{i t H_{S}} e^{-i k \cdot q}=\exp \left\{i t\left(\left[e^{i k \cdot q} p e^{-i k \cdot q}\right]^{2 / 2}+V(q)\right\}=\exp i t\left\{(p+k)^{2 / 2}+V(q)\right\}\right.
$$

and the right-hand side of (2.9) is equal to the right-hand side of (2.8).

Using Lemma 2.1 the quantity (2.7) becomes

$$
\begin{aligned}
\lambda^{2} \int_{0}^{t / \lambda^{2}} d t_{1} \int_{0}^{s / \lambda^{2}} d t_{2} \int_{\mathbb{R}^{d}} \overline{\left(S_{t_{1}} f\right)}(k)\left(S_{t_{2}} g\right)(k) d k \\
\quad e^{i t_{1} H_{S}}(-i p) e^{i\left(t_{2}-t_{1}\right)\left(H_{S}+p \cdot k\right)}(i p) e^{-i t_{2} H_{S}} e^{i\left(t_{2}-t_{1}\right)|k|^{2}}
\end{aligned}
$$

and, by the change of variables:

$$
\lambda^{2} t_{h}=s_{h}, \quad h=1,2 ; \quad \tau_{1}=s_{1}, \quad\left(s_{2}-s_{1}\right) / \lambda^{2}=\tau_{2},
$$

(2.10) becomes

$$
\begin{aligned}
& \int_{0}^{t} d \tau_{1} \int_{-\tau_{1} / \lambda^{2}}^{\left(s-\tau_{1}\right) / \lambda^{2}} d \tau_{2} \int_{\mathbb{R}^{d}} \bar{f}(k)\left(S_{\tau_{2}} g\right)(k) d k \\
& e^{i \tau_{1} H_{S} / \lambda^{2}}(-i p) e^{i \tau_{2}\left(H_{S}+p \cdot k\right)}(i p) e^{-i \tau_{1} H_{S} / \lambda^{2}} e^{-i \tau_{2} H_{S}} e^{i \tau_{2}|k|^{2}}
\end{aligned}
$$

The following lemma describes the commutation relations of $e^{i \tau_{1} H_{S}}$. 
LEMMA 2.2. For any bounded function or polynomial $F$,

$$
\left[e^{i t H_{S}}, F(p)\right]=-i \int_{0}^{t} d s e^{i s H_{S}}[F(p), V(q)] e^{i(t-s) H_{S}} .
$$

Proof: Define

$$
X(t):=\left[e^{i t H_{S}}, F(p)\right], \quad Y(t):=-i \int_{0}^{t} d s e^{i s H_{S}}[F(p), V(q)] e^{i(t-s) H_{S}},
$$

then

$$
X(0)=\cdot Y(0)=0
$$

and

$$
\frac{d}{d t} X(t)=i X(t) H_{S}-i e^{i t H_{S}}[F(p), V(q)]
$$

On the other hand,

$$
\begin{aligned}
\frac{d}{d t} Y(t) & =-i e^{i t H_{S}}[F(p), V(q)]-i^{2} \int_{0}^{t} d s e^{i s H_{S}}[F(p), V(q)] e^{i(t-s) H_{S}} H_{S} \\
& =i Y(t) H_{S}-i e^{i t H_{S}}[F(p), V(q)] .
\end{aligned}
$$

and this implies the thesis.

An application of Lemma 2.2 to the case $F(p)=p$, which gives $[p, V]=-i V^{\prime}$, shows that (2.10) is equal to

$$
\begin{aligned}
\int_{0}^{t} d \tau_{1} \int_{-\tau_{1} / \lambda^{2}}^{\left(s-\tau_{1}\right) / \lambda^{2}} d \tau_{2} \int_{\mathbb{R}^{d}} \bar{f}(k)\left(S_{\tau_{2}} g\right)(k) \times \\
\times\left(p-i \int_{0}^{\tau_{1} / \lambda^{2}} d s e^{i s H_{S}} V^{\prime}(q) e^{-i s H_{S}}\right) e^{i \tau_{1} H_{S} / \lambda^{2}} e^{i \tau_{2}\left(H_{S}+p \cdot k\right)} \times \\
\quad \times e^{-i\left(\tau_{1} / \lambda^{2}+\tau_{2}\right) H_{S}}\left(p+i \int_{0}^{-\tau_{1} / \lambda^{2}-\tau_{2}} d s e^{i s H_{S}} V^{\prime}(q) e^{-i s H_{S}}\right) e^{i \tau_{2}|k|^{2}}
\end{aligned}
$$

It is known (cf. [7]) that the limit

$$
\lim _{\lambda \rightarrow 0} \int_{0}^{r / \lambda^{2}} d s e^{i s H_{S}} V^{\prime}(q) e^{-i s H_{S}}=: \Gamma_{H_{S}}\left(V^{\prime}(q)\right)
$$

exists for any $r>0$ and $V^{\prime} \in \mathbf{L}^{1}\left(\mathbb{R}^{d}\right) \cap \mathbf{L}^{2}\left(\mathbb{R}^{d}\right)$. Thus (2.17) is equal to

$$
\begin{aligned}
o(1) & +\int_{0}^{t} d \tau_{1} \int_{-\tau_{1} / \lambda^{2}}^{\left(s-\tau_{1}\right) / \lambda^{2}} d \tau_{2} \int_{\mathbb{R}^{d}} \bar{f}(k)\left(S_{\tau_{2}} g\right)(k) \times \\
& \times\left(p-i \Gamma_{H_{S}}\left(V^{\prime}(q)\right)\right) e^{i \tau_{1} H_{S} / \lambda^{2}} e^{i \tau_{2}\left(H_{S}+p \cdot k\right)} e^{-i\left(\tau_{1} / \lambda^{2}+\tau_{2}\right) H_{S}}\left(p-i \Gamma_{H_{S}}\left(V^{\prime}(q)\right)\right)^{*} e^{i \tau_{2}|k|^{2}},
\end{aligned}
$$


where and hereinafter, by $o(1)$ we denote a quantity which tends to zero as $\lambda \rightarrow 0$. From usual scattering theory, for $V \in \mathbf{L}^{1}\left(\mathbb{R}^{d}\right) \cap \mathbf{L}^{2}\left(\mathbb{R}^{d}\right)$ and any $r>0$, the limits

$$
\begin{aligned}
& \Omega\left( \pm H_{S}, \mp p^{2}\right):=s-\lim _{\lambda \rightarrow 0} e^{ \pm i r H_{S} / \lambda^{2}} e^{\mp i r p^{2} / \lambda^{2}}, \\
& \Omega\left( \pm p^{2}, \mp H_{S}\right):=s-\lim _{\lambda \rightarrow 0} e^{ \pm i r p^{2} / \lambda^{2}} e^{\mp i r H_{S} / \lambda^{2}}
\end{aligned}
$$

exist and are called the $\mathrm{M} \phi$ ller wave operator. Moreover, all of them are complete.

With these notation, one can rewrite (2.19) as

$$
\begin{aligned}
o(1)+\int_{0}^{t} d \tau_{1} \int_{-\tau_{1} / \lambda^{2}}^{\left(s-\tau_{1}\right) / \lambda^{2}} d \tau_{2} \int_{\mathbb{R}^{d}} \bar{f}(k)\left(S_{\tau_{2}} g\right)(k) \\
\left(p-i \Gamma_{H_{S}}\left(V^{\prime}(q)\right)\right) \Omega\left(+H_{S},-p^{2}\right) e^{i \tau_{1} p^{2} / \lambda^{2}} e^{i \tau_{2}\left(H_{S}+p \cdot k\right)} e^{-i \tau_{1} p^{2} / \lambda^{2}} \\
\Omega^{*}\left(+H_{S},-p^{2}\right) e^{-i \tau_{2} H_{S}}\left(p-i \Gamma_{H_{S}}\left(V^{\prime}(q)\right)\right)^{*} e^{i \tau_{2}|k|^{2}} .
\end{aligned}
$$

Now we have to investigate the operator

$$
e^{i \tau_{1} p^{2} / \lambda^{2}} e^{i \tau_{2}\left(H_{S}+p \cdot k\right)} e^{-i \tau_{1} p^{2} / \lambda^{2}}=\exp i \tau_{2}\left(p^{2}+e^{i \tau_{1} p^{2} / \lambda^{2}} V(q) e^{-i \tau_{1} p^{2} / \lambda^{2}}+p \cdot k\right) .
$$

Since

$$
e^{i n p^{2}} V(q) e^{-i n p^{2}}=V(q+n p)
$$

we know that the right-hand side of (2.23) is equal to

$$
e^{i \tau_{2}\left(p^{2}+p \cdot k+V\left(q+\tau_{1} p / \lambda^{2}\right)\right)}
$$

and since $V(x) \rightarrow 0$ as $|x| \rightarrow \infty$, it is intuitively clear that the quantity (2.25) converges to $\exp \left(i \tau_{2}\left(p^{2}+p \cdot k\right)\right)$ as $\lambda \rightarrow 0$. The following lemma shows that this intuition is correct.

LEMMA 2.3. Define the new operator

$$
Y_{n}(t):=e^{i t\left(p^{2}+p \cdot k+V(q+n p)\right)} e^{-i t\left(p^{2}+p \cdot k\right)} .
$$

If the potential function $V$ is in the Schwartz class, one has

$$
s-\lim _{n \rightarrow \infty} Y_{n}(t)=1 .
$$

Proof: It is easy to verify that

$$
\begin{gathered}
Y_{n}(t)=1+\int_{0}^{t} d t_{1} V\left(q+\left(n+t_{1}\right) p+t_{1} k\right)+\int_{0}^{t} d t_{1} \int_{0}^{t_{1}} d t_{2} \\
V\left(q+\left(n+t_{1}\right) p+t_{1} k\right) V\left(q+\left(n+t_{2}\right) p+t_{2} k\right)+\ldots \\
\quad+\int_{0}^{t} d t_{1} \int_{0}^{t_{1}} d t_{2} \ldots \int_{0}^{t_{N-1}} d t_{N} \\
V\left(q+\left(n+t_{1}\right) p+t_{1} k\right) V\left(q+\left(n+t_{2}\right) p+t_{2} k\right) \ldots V\left(q+\left(n+t_{N}\right) p+t_{N} k\right)+\ldots
\end{gathered}
$$


Since $V$ is bounded, in order to prove (2.27), it is enough to show that for any $N \geq 1$,

$$
s-\lim _{n \rightarrow \infty} V\left(q+\left(n+t_{1}\right) p+k_{1}\right) V\left(q+\left(n+t_{2}\right) p+k_{2}\right) \ldots V\left(q+\left(n+t_{N}\right) p+k_{N}\right)=0 .
$$

By Fourier transform, one obtains that, writing for convenience $k_{h}:=t_{h} k$,

$$
\begin{gathered}
V\left(q+\left(n+t_{1}\right) p+k_{1}\right) V\left(q+\left(n+t_{2}\right) p+k_{2}\right) \ldots V\left(q+\left(n+t_{N}\right) p+k_{N}\right) \\
=\int_{\mathbb{R}^{N d}} \hat{V}\left(x_{1}\right) \ldots \hat{V}\left(x_{N}\right) e^{i\left[\sum_{1 \leq h \leq N}\left|x_{h}\right|^{2}+\sum_{1 \leq \alpha<\beta \leq N} x_{\alpha} \cdot x_{\beta}\right]} \\
e^{i \sum_{1 \leq h \leq N}\left(k_{h} \cdot x_{h}+t_{h}\left|x_{h}\right|^{2}\right)+i \sum_{1 \leq \alpha<\beta \leq N} x_{\alpha} \cdot x_{\beta} t_{\alpha}} e^{i p \cdot \sum_{1 \leq h \leq N} x_{h}\left(n+t_{h}\right)} e^{i q \cdot \sum_{1 \leq h \leq N} x_{h}} .
\end{gathered}
$$

The Riemann-Lebesgue lemma gives our claim.

By using these results we are able to rewrite (2.22) as

$$
\begin{aligned}
& o(1)+\int_{0}^{t} d \tau_{1} \int_{-\tau_{1} / \lambda^{2}}^{\left(s-\tau_{1}\right) / \lambda^{2}} d \tau_{2} \int_{\mathbb{R}^{d}} \bar{f}(k)\left(S_{\tau_{2}} g\right)(k)\left(p-i \Gamma_{H_{S}}\left(V^{\prime}(q)\right)\right) \\
& \Omega\left(+H_{S},-p^{2}\right) e^{i \tau_{2}\left(p^{2}+p \cdot k\right)} \Omega^{*}\left(+H_{S},-p^{2}\right) e^{-i \tau_{2} H_{S}}\left(p-i \Gamma_{H_{S}}\left(V^{\prime}(q)\right)\right)^{*} e^{i \tau_{2}|k|^{2}}
\end{aligned}
$$

which tends, as $\lambda \rightarrow 0$, to

$$
\begin{aligned}
& \left\langle\chi_{[0, t)}, \chi_{[0, s)}\right) \int_{-\infty}^{\infty} d t_{1} \int_{\mathbb{R}^{d}} \bar{f}(k)\left(S_{t_{1}} g\right)(k)\left(p-i \Gamma_{H_{S}}\left(V^{\prime}(q)\right)\right) \\
& \Omega\left(+H_{S},-p^{2}\right) e^{i t_{1}\left(p^{2}+p \cdot k\right)} \Omega^{*}\left(+H_{S},-p^{2}\right) e^{-i t_{1} H_{S}}\left(p-i \Gamma_{H_{S}}\left(V^{\prime}(q)\right)\right)^{*} e^{i t_{2}|k|^{2}} .
\end{aligned}
$$

The intertwining property of the scattering operator implies that

$$
\Omega^{*}\left(+H_{S},-p^{2}\right) e^{-i t_{1} H_{S}}=e^{-i t_{1} p^{2}} \Omega^{*}\left(+H_{S},-p^{2}\right),
$$

so (2.32) becomes

$$
\begin{aligned}
& \left\langle\chi_{[0, t)}, \chi_{[0, s)}\right) \int_{-\infty}^{\infty} d t_{1} \int_{\mathbb{R}^{d}} \bar{f}(k)\left(S_{t_{1}} g\right)(k) \\
& \left(p-i \Gamma_{H_{S}}\left(V^{\prime}(q)\right)\right) \Omega\left(+H_{S},-p^{2}\right) e^{i t_{1} p \cdot k} \Omega^{*}\left(+H_{S},-p^{2}\right)\left(p-i \Gamma_{H_{S}}\left(V^{\prime}(q)\right)\right)^{*} e^{i t_{2}|k|^{2}}
\end{aligned}
$$

Finally, Lemma 2.2 shows that

$$
\left.p \Omega\left(+H_{S},-p^{2}\right)=\Omega\left(+H_{S},-p^{2}\right) p+i \Gamma_{H_{S}}\left(V^{\prime}(q)\right)\right) \Omega\left(+H_{S},-p^{2}\right),
$$

therefore the two-point function (2.6) has the following limit

$$
\left\langle\chi_{[0, t)}, \chi_{[0, s)}\right\rangle \int_{-\infty}^{\infty} d t_{1} \int_{\mathbb{R}^{d}} \bar{f}(k)\left(S_{t_{1}} g\right)(k) \Omega\left(+H_{S},-p^{2}\right) p e^{i t_{1} p \cdot k} p \Omega^{*}\left(+H_{S},-p^{2}\right) e^{i t_{1}|k|^{2}}
$$


In order to understand which type of quantum noise can appear in our limit, let us examine the four-point functions. Vanishing of the crossing diagrams is proved with the same technique as in [1] (Section (12.19)) or [3]. Therefore, we have to consider only those four-point functions which correspond to products of the creation and annihilation operators of the following types (noncrossing diagrams)

$$
a a^{+} a a^{+}, \quad a a a^{+} a^{+} \text {, }
$$

respectively. That is, we must consider the limit of

$$
\begin{gathered}
\Delta(\lambda, 0101):=\lambda^{4} \int_{0}^{T_{1} / \lambda^{2}} d t_{1} \int_{0}^{T_{2} / \lambda^{2}} d t_{2} \int_{0}^{T_{3} / \lambda^{2}} d t_{3} \int_{0}^{T_{4} / \lambda^{2}} d t_{4} \\
\langle 0| \int_{\mathbb{R}^{4 d}} d k_{1} d k_{2} d k_{3} d k_{4} a_{k_{1}} \overline{\left(S_{t_{1}} f_{1}\right)}\left(k_{1}\right) a_{k_{2}}^{+}\left(S_{t_{2}} f_{2}\right)\left(k_{2}\right) a_{k_{3}} \overline{\left(S_{t_{3}} f_{3}\right)\left(k_{3}\right) a_{k_{4}}^{+}\left(S_{t_{4}} f_{4}\right)\left(k_{4}\right)|0\rangle} \\
e^{i t_{1} H_{S}}(-i p) e^{i k_{1} \cdot q} e^{-i t_{1} H_{S}} \cdot e^{i t_{2} H_{S}} e^{-i k_{2} \cdot q}(i p) e^{-i t_{2} H_{S}} \\
\cdot e^{i t_{3} H_{S}}(-i p) e^{i k_{3} \cdot q} e^{-i t_{3} H_{S}} \cdot e^{i t_{4} H_{S}} e^{-i k_{4} \cdot q}(i p) e^{-i t_{4} H_{S}}
\end{gathered}
$$

and

$$
\begin{gathered}
\Delta(\lambda, 0011):=\lambda^{4} \int_{0}^{T_{1} / \lambda^{2}} d t_{1} \int_{0}^{T_{2} / \lambda^{2}} d t_{2} \int_{0}^{T_{3} / \lambda^{2}} d t_{3} \int_{0}^{T_{4} / \lambda^{2}} d t_{4} \\
\left\langle 0\left|\int_{\mathbb{R}^{4 d}} d k_{1} d k_{2} d k_{3} d k_{4} a_{k_{1}} \overline{\left(S_{t_{1}} f_{1}\right)}\left(k_{1}\right) a_{k_{2}} \overline{\left(S_{t_{2}} f_{2}\right)}\left(k_{2}\right) a_{k_{3}}^{+}\left(S_{t_{3}} f_{3}\right)\left(k_{3}\right) a_{k_{4}}^{+}\left(S_{t_{4}} f_{4}\right)\left(k_{4}\right)\right| 0\right\rangle \\
e^{i t_{1} H_{S}}(-i p) e^{i k_{1} \cdot q} e^{-i t_{1} H_{S}} \cdot e^{i t_{2} H_{S}}(-i p) e^{i k_{2} \cdot q} e^{-i t_{2} H_{S}} \\
\cdot e^{i t_{3} H_{S}} e^{-i k_{3} \cdot q}(i p) e^{-i t_{3} H_{S}} \cdot e^{i t_{4} H_{S}} e^{-i k_{4} \cdot q}(i p) e^{-i t_{4} H_{S}}
\end{gathered}
$$

The Gaussian form implies that

$$
\begin{gathered}
\Delta(\lambda, 0101):=\lambda^{4} \int_{0}^{T_{1} / \lambda^{2}} d t_{1} \int_{0}^{T_{2} / \lambda^{2}} d t_{2} \int_{0}^{T_{3} / \lambda^{2}} d t_{3} \int_{0}^{T_{4} / \lambda^{2}} d t_{4} \\
\int_{\mathbb{R}^{2 d}} d k_{1} d k_{3} \overline{\left(S_{t_{1}} f_{1}\right)}\left(k_{1}\right)\left(S_{t_{2}} f_{2}\right)\left(k_{1}\right) \overline{\left(S_{t_{3}} f_{3}\right)}\left(k_{3}\right)\left(S_{t_{4}} f_{4}\right)\left(k_{3}\right) \\
e^{i t_{1} H_{S}}(-i p) e^{i k_{1} \cdot q} e^{-i t_{1} H_{S}} \cdot e^{i t_{2} H_{S}} e^{-i k_{1} \cdot q}(i p) e^{-i t_{2} H_{S} .} \\
\cdot e^{i t_{3} H_{S}}(-i p) e^{i k_{3} \cdot q} e^{-i t_{3} H_{S}} \cdot e^{i t_{4} H_{S}} e^{-i k_{3} \cdot q}(i p) e^{-i t_{4} H_{S}},
\end{gathered}
$$

which practically is a product of two objects both similar to the quantity (2.6). Therefore the same argument used to prove (2.6) gives the following result.

LEMMA 2.4. If the potential function $V$ is in the Schwartz class, the limit, as $\lambda \rightarrow 0$, of the term $\Delta(\lambda, 0101)$ is equal to

$$
\begin{gathered}
\left\langle\chi_{\left[0, T_{1}\right)}, \chi_{\left[0, T_{2}\right)}\right)\left\langle\chi_{\left[0, T_{3}\right)}, \chi_{\left[0, T_{4}\right)}\right\rangle \int_{-\infty}^{\infty} d t \int_{-\infty}^{\infty} d s \int_{\mathbb{R}^{2 d}} d k_{1} d k_{2} \overline{f_{1}}\left(k_{1}\right)\left(S_{t} f_{2}\right)\left(k_{1}\right) \overline{f_{3}}\left(k_{2}\right) \\
\left(S_{s} f_{4}\right)\left(k_{2}\right) \Omega\left(+H_{S},-p^{2}\right) p^{2} e^{i t p \cdot k_{1}} e^{i s p \cdot k_{2}} p^{2} \Omega^{*}\left(+H_{S},-p^{2}\right) e^{i t\left|k_{1}\right|^{2}} e^{i s\left|k_{2}\right|^{2}}
\end{gathered}
$$


Proof: In the investigation of the two-point function, the operator $p$ behaves like a bounded operator if one takes on the system space the partial expectation of (2.37) by a linear function of the form $\langle\xi, \cdot \eta\rangle$ with $\xi, \eta$ in the Schwartz class. Because of this, one can repeat in this case the proof for the two-point function (2.6) and this leads to the desired conclusion.

Now we are going to investigate the limit of the term $\Delta(\lambda, 0101)$ and in the following discussion we shall always assume, without any further explicit mention of this fact, that the potential function is in the Schwartz class.

LEMMA 2.5. If the potential function $V$ is in the Schwartz class, the limit, as $\lambda \rightarrow 0$, of the term $\Delta(\lambda, 0011)$, i.e. (2.36), is equal to

$$
\begin{array}{r}
\left\langle\chi_{\left[0, T_{1}\right)}, \chi_{\left[0, T_{4}\right)}\right\rangle\left\langle\chi_{\left[0, T_{2}\right)}, \chi_{\left[0, T_{3}\right)}\right\rangle \int_{-\infty}^{\infty} d t \int_{-\infty}^{\infty} d s \int_{\mathbb{R}^{2 d}} d k_{1} d k_{2} \overline{f_{1}}\left(k_{1}\right) \\
\left(S_{t} f_{4}\right)\left(k_{1}\right) \overline{f_{2}}\left(k_{2}\right)\left(S_{S} f_{3}\right)\left(k_{2}\right) \Omega\left(+H_{S},-p^{2}\right) p\left(p+k_{1}\right) e^{i t p \cdot k_{1}} \\
e^{i s p \cdot k_{2}}\left(p+k_{1}\right) p \Omega^{*}\left(+H_{S},-p^{2}\right) e^{i t\left|k_{1}\right|^{2}} e^{i s\left(\left|k_{2}\right|^{2}+k_{1} \cdot k_{2}\right)}
\end{array}
$$

Proof: Because of the Gaussian form, (2.36) is equal to

$$
\begin{aligned}
& \Delta(\lambda, 0011):=\lambda^{4} \int_{0}^{T_{1} / \lambda^{2}} d t_{1} \int_{0}^{T_{2} / \lambda^{2}} d t_{2} \int_{0}^{T_{3} / \lambda^{2}} d t_{3} \int_{0}^{T_{4} / \lambda^{2}} d t_{4} \\
& \int_{\mathbb{R}^{2 d}} d k_{1} d k_{2} \overline{\left(S_{t_{1}} f_{1}\right)}\left(k_{1}\right) \overline{\left(S_{t_{2}} f_{2}\right)}\left(k_{2}\right)\left(S_{t_{3}} f_{3}\right)\left(k_{2}\right)\left(S_{t_{4}} f_{4}\right)\left(k_{1}\right) \\
& e^{i t_{1} H_{S}}(-i p) e^{i k_{1} \cdot q} e^{-i t_{1} H_{S}} \cdot e^{i t_{2} H_{S}}(-i p) e^{i k_{2} \cdot q} e^{-i t_{2} H_{S}} . \\
& \cdot e^{i t_{3} H_{S}} e^{-i k_{2} \cdot q}(i p) e^{-i t_{3} H_{S}} \cdot e^{i t_{4} H_{S}} e^{-i k_{1} \cdot q}(i p) e^{-i t_{4} H_{S}} .
\end{aligned}
$$

Now it follows from Lemma 2.1 that the product of the operators in (2.40) becomes

$$
\begin{aligned}
& e^{i t_{1} H_{S}}(-i p) e^{-i t_{1}\left(H_{S}+p \cdot k_{1}\right)} \cdot e^{i t_{2}\left(H_{S}+p \cdot k_{1}\right)}\left(-i\left(p+k_{1}\right)\right) e^{-i t_{2}\left(H_{S}+p \cdot\left(k_{1}+k_{2}\right)\right)} e^{i t_{3}\left(H_{S}+p \cdot\left(k_{1}+k_{2}\right)\right)} \\
& \quad\left(i\left(p+k_{1}\right)\right) e^{-i t_{3}\left(H_{S}+p \cdot k_{1}\right)} e^{i t_{4}\left(H_{S}+p \cdot k_{1}\right)}(i p) e^{-i t_{4} H_{S}} e^{i\left(t_{4}-t_{1}\right)\left|k_{1}\right|^{2}} e^{i\left(t_{3}-t_{2}\right)\left(\left|k_{2}\right|^{2}+k_{1} \cdot k_{2}\right)}
\end{aligned}
$$

And again the result follows from the same arguments used to find the limit of the two-point function.

\section{The limit Fock module}

In Section 2, we have investigated the limit behaviour of the two- and four-point functions. That discussion suggested that the limit of the evolution must be related to the M $\phi$ ller wave operator and that it should live on some Hilbert module.

In this section we shall investigate the structure of this limit Hilbert module and prove that it differs from the one found in the case with $V=0$ (cf. [3]). In particular, it is a Hilbert module over the Abelian $*$-algebra (not a $C^{*}$-algebra) 
which is the image, by the automorphism implemented by the wave operator, of the algebra of all the polynomials in the momentum operator $p$ and its bounded measurable functions. More precisely, denote by $\mathfrak{P}_{n}$ the set of all polynomials (of one variable) of degree $n$ and define

$$
\mathcal{P}_{\Omega}:=\left\{\Omega\left(H_{S},-p^{2}\right) P_{n}(p) e^{i t p \cdot k} \Omega^{*}\left(H_{S},-p^{2}\right) \mid n \in \mathbb{N}, \quad k \in \mathbb{R}^{d}, \quad P_{n} \in \mathfrak{P}_{n}\right\} .
$$

It is clear that $\mathcal{P}_{\Omega}$ is a $*$-algebra admitting a dense subset of $\mathbf{L}^{2}\left(\mathbb{R}^{d}\right)$ as invariant domain. For each $f \in \mathcal{S}\left(\mathbb{R}^{n}\right)$ define

$$
\tilde{f}_{P_{n}}(t):=\int_{\mathbb{R}^{d}} d k \Omega\left(H_{S},-p^{2}\right) P_{n}(p) e^{i t p \cdot k} \Omega^{*}\left(H_{S},-p^{2}\right)\left(S_{t} f\right)(k)
$$

and

$$
\mathcal{F}:=\left\{\tilde{f}_{a} \mid f \in \mathcal{S}\left(\mathbb{R}^{n}\right), \quad a \text { is a polynomial in } p\right\} .
$$

It is easy to show that the elements of $\mathcal{F}$ are operators on $\mathcal{H}_{S}$, defined on the same domain as $\mathcal{P}_{\Omega}$ and that $\mathcal{F}$ is a two-sided $\mathcal{P}_{\Omega}$-module. On the $\mathcal{P}_{\Omega}$-module $\mathcal{F}$ introduce the $\mathcal{P}_{\Omega}$-valued inner product $(\cdot \mid \cdot)$ :

$$
\left(\tilde{f}_{a} \mid \tilde{g}_{b}\right):=\int_{\mathbb{R}^{d}} \int_{\mathbb{R}} d t \Omega\left(H_{S},-p^{2}\right) a^{*}(p) b(p) e^{i t p \cdot k} \Omega^{*}\left(H_{S},-p^{2}\right) \bar{f}(k)\left(S_{t} g\right)(k),
$$

and so $(\mathcal{F} /(\cdot \mid \cdot),(\cdot \mid \cdot))$ is a (pre)Hilbert module and in the following we shall denote the associated Hilbert module by $\mathcal{F}$.

Starting from this Hilbert module, let us consider the algebraic tensor product $\mathbf{L}^{2}\left(\mathbb{R}_{+}\right) \odot \mathcal{F}$ on which we introduce the inner product

$$
\left(\alpha \odot \tilde{f}_{a} \mid \beta \odot \tilde{g}_{b}\right):=\langle\alpha, \beta\rangle_{\mathbf{L}^{2}\left(\mathbb{R}_{+}\right)} \cdot\left(\tilde{f}_{a} \mid \tilde{g}_{b}\right) .
$$

Thus we get a new Hilbert module. This new Hilbert module is the basic object by means of which we can define our limit quantum stochastic process. For each given $n \in \mathbb{N}$, on the algebraic tensor product $\left(\mathbf{L}^{2}\left(\mathbb{R}_{+}\right) \odot \mathcal{F}\right)^{\odot n}$ we introduce the inner product

$$
\begin{gathered}
\left(\alpha_{1} \odot \tilde{f}_{1, a_{1}} \odot \cdots \odot \alpha_{n} \odot \tilde{f}_{n, a_{n}} \mid \beta_{1} \odot \tilde{g}_{1, b_{1}} \odot \cdots \odot \beta_{n} \odot \tilde{g}_{n, b_{n}}\right) \\
:=\prod_{h=1}^{n}\left\langle\alpha_{h}, \quad \beta_{h}\right\rangle_{\mathbf{L}^{2}\left(\mathbb{R}_{+}\right)} \cdot \int_{\mathbb{R}^{n}} d u_{1} \cdots d u_{n} \int_{\mathbb{R}^{n d}} d k_{1} \cdots d k_{n} \exp \left(i \sum_{1 \leq r \leq h \leq n-1} u_{r} k_{r} k_{h+1}\right) \\
\Omega\left(H_{S},-p^{2}\right) \prod_{h=1}^{n}\left[a_{h}^{*}\left(p+\sum_{j=1}^{h-1} k_{j}\right) b_{h}\left(p+\sum_{j=1}^{h-1} k_{j}\right) e^{-i u_{h} k_{h} \cdot p} \bar{f}_{h}\left(k_{h}\right)\left(S_{u_{h}} g_{h}\right)\left(k_{h}\right)\right] \Omega^{*}\left(H_{S},-p^{2}\right)^{*},
\end{gathered}
$$

where the equivalence classes are defined in a similar way as in the case $n=1$ and, by definition, the summation is extended to the set of indices $1 \leq r \leq h \leq n-1$, with $n=1$ put equal to zero. 
Thus for each $n \in \mathbb{N}$, with the $\mathcal{P}_{\Omega}$-right sesquilinear, $\mathcal{P}_{\Omega}$-valued, form given by (3.6), $\left(\mathbf{L}^{2}(\mathbb{R}) \odot \mathcal{F}\right)^{\odot n}$ becomes a right $\mathcal{P}_{\Omega}$-pre-Hilbert module and the symbol $\left(\mathbf{L}^{2}(\mathbb{R}) \odot \mathcal{F}\right)^{\otimes n}$ will be used to denote it.

Since for each $n \in \mathbb{N},\left(\mathbf{L}^{2}(\mathbb{R}) \odot \mathcal{F}\right)^{\otimes n}$ is a $\mathcal{P}_{\Omega}$-pre-Hilbert module, the direct sum $\mathbf{C} \oplus \bigoplus_{n=1}^{\infty}\left(\mathbf{L}^{2}(\mathbb{R}) \odot \mathcal{F}\right)^{\otimes n}$ makes sense and will be denoted by $\Gamma\left(\mathbf{L}^{2}\left(\mathbb{R}_{+}\right) \odot \mathcal{F}\right)$ and called the $\mathcal{P}_{\Omega}$-free Fock module over $\mathbf{L}^{2}\left(\mathbb{R}_{+}\right) \odot \mathcal{F}$. In this pre-Hilbert module, the vector $\Psi:=1 \oplus 0 \oplus 0 \cdots$ is called the vacuum vector.

LEMMA 3.1. The number vector subset

$$
\Gamma:=\left\{\left(\alpha_{1} \odot \tilde{f}_{1}\right) \otimes \cdots \otimes\left(\alpha_{n} \odot \tilde{f}_{n}\right) \Psi \mid n \in \mathbb{N}, \alpha_{j} \in \mathbf{L}^{2}\left(\mathbb{R}_{+}\right), \tilde{f}_{j} \in \mathcal{F}, j=1, \ldots, n\right\}
$$

is a $\mathcal{P}_{\Omega}$-total subset of $\Gamma\left(\mathbf{L}^{2}\left(\mathbb{R}_{+}\right) \odot \mathcal{F}\right)$.

Proof: It is clear just by using the definition of Hilbert module.

Definition 3.1. For each element of $\mathbf{L}^{2}\left(\mathbb{R}_{+}\right) \odot \mathcal{F}$, the creator with respect to this element, denoted by $A^{+}(\cdot)$, is defined on the $\mathcal{P}_{\Omega^{2}}$-right linear span of $\Gamma$ by $\mathcal{P}_{\Omega}$-right linearity and

$$
A^{+}(\alpha \odot \tilde{f})\left[\left(\alpha_{1} \odot \tilde{f}_{1}\right) \otimes \cdots \otimes\left(\alpha_{n} \odot \tilde{f}_{n}\right) \Psi\right]:=(\alpha \odot \tilde{f}) \otimes\left(\alpha_{1} \odot \tilde{f}_{1}\right) \otimes \cdots \otimes\left(\alpha_{n} \odot \tilde{f}_{n}\right) \Psi,
$$

where $n \in \mathbb{N}, \alpha, \alpha_{j} \in L^{2}\left(\mathbb{R}_{+}\right), \tilde{f}, \tilde{f}_{j} \in \mathcal{F}, j=1, \ldots, n$.

The annihilation operator $A(\cdot)$ is defined as the adjoint of the creation operator on the domain of number vectors. $\left.\tilde{f}_{1}\right)$.

REMARK. In general, $A^{+}\left(\alpha_{1} \odot \tilde{f}_{1}\right) A^{+}\left(\alpha_{2} \odot \tilde{f}_{2}\right)$ is not equal to $A^{+}\left(\alpha_{2} \odot \tilde{f}_{2}\right) A^{+}\left(\alpha_{1} \odot\right.$

The above discussion suggests us to define the collective module creator

$$
A_{\lambda}^{+}(S, T, f, a):=\lambda \int_{S / \lambda^{2}}^{T / \lambda^{2}} d t \int_{\mathbb{R}^{d}} d k e^{-i t H_{s}} e^{i k \cdot p} a(p) e^{i t H_{S}} \otimes\left(S_{t} f\right)(k) a_{k}^{+},
$$

where $0 \leq S \leq T<+\infty, f \in \mathcal{S}\left(\mathbb{R}^{d}\right)$ and $a(p)$ is a polynomial in $p$.

THEOREM 3.1. For any $N, N^{\prime} \in \mathbb{N}$, the limit of

$$
\left\langle\prod_{h=1}^{N} A_{\lambda}^{+}\left(S_{h}, T_{h}, f_{h}, a_{h}\right) \Phi, \prod_{h=1}^{N^{\prime}} A_{\lambda}^{+}\left(S_{h}^{\prime}, T_{h}^{\prime}, f_{h}^{\prime}, a_{h}^{\prime}\right) \Phi\right\rangle
$$

exists and is equal to

$$
\left\langle\prod_{h=1}^{N} A^{+}\left(\chi_{\left[S_{h}, T_{h}\right]} \odot \tilde{f}_{h, a_{h}}\right) \Psi, \prod_{h=1}^{N^{\prime}} A^{+}\left(\chi_{\left[S_{h}^{\prime}, T_{h}^{\prime}\right]} \odot \tilde{f}_{h, a_{h}^{\prime}}^{\prime}\right) \Psi\right\rangle .
$$

Proof: The theorem is proved by the same arguments as used in the proof of Lemma 2.5 . 
By the same techniques as used in [3], one can prove that the quantum noise is of free type in the sense that only the noncrossing diagrams contribute to its correlation functions. Therefore our Hilbert module is a nonlinear deformation of the usual free (or Boltzmannian) Fock module. The stochastic calculus over the free Fock module has been first studied (cf. [10]).

\section{The limit stochastic process}

Now we compute the vacuum statistics of the creation and annihilation fields on the limit Hilbert module, i.e. the expectation values

$$
\left\langle\Psi, A^{\varepsilon(1)}\left(\alpha_{1} \odot f_{1}\right) \cdots A^{\varepsilon(2 n)}\left(\alpha_{2 n} \odot f_{2 n}\right) \Psi\right\rangle,
$$

where $n \in \mathbb{N}, \alpha_{j} \in \mathbf{L}^{2}\left(\mathbb{R}_{+}\right), f_{j} \in \mathcal{F}(j=1, \ldots, 2 n), \varepsilon \in\{0,1\}^{2 n}$ and

$$
A^{0}:=A, \quad A^{1}:=A^{+},
$$

and where for simplicity we shall not distinguish between $f$ and $\tilde{f}$ (e.g. we restrict ourselves to real-valued test functions). Moreover, it is necessary to know (4.1) only in the case of

$$
\varepsilon(1)=0, \quad \varepsilon(2 n)=1 .
$$

LEMMA 4.1. The inner product (4.1) is not equal to zero only if

$$
\sum_{h=1}^{2 n} \varepsilon(h)=n .
$$

Proof: This is a standard fact on free Fock modules.

Let be given a subset $\left\{m_{h}\right\}_{h=1}^{n} \subset\{1, \ldots, 2 n\}$ with $1<m_{1}<\ldots<m_{n}=2 n$. We say that $\left\{m_{h}\right\}_{h=1}^{n}$ admits a noncrossing pair partition if denoting by $\left\{m_{h}^{\prime}\right\}_{h=1}^{n}$ an ordered version of the set $\{1, \ldots, 2 n\} \backslash\left\{m_{h}\right\}_{h=1}^{n}$, the family $\left\{m_{h}^{\prime}, m_{h}\right\}_{h=1}^{n}$ is a noncrossing pair partition of $\{1, \ldots, 2 n\}$. By [3], if this is the case, then the choice of the $\left\{m_{h}^{\prime}\right\}$ is unique.

For a given $\varepsilon \in\{0,1\}^{2 n}$ denote

$$
\left\{m_{h}\right\}_{h=1}^{n}:=\{r \in\{1, \ldots, 2 n\} \mid \varepsilon(r)=1\}, \quad 1<m_{1}<\ldots<m_{n}=2 n .
$$

We say that $\varepsilon \in\{0,1\}^{2 n}$ admits a noncrossing pair partition of $\{1, \ldots, 2 n\}$ if $\left\{m_{h}\right\}_{h=1}^{n}$ does.

LEMMA 4.2. The inner product (4.1) is equal to zero if $\varepsilon$ does not admit a noncrossing pair partition of $\{1, \ldots, 2 n\}$.

Now we are led to consider the expression (4.1) when $\varepsilon$ admits a (unique) noncrossing pair partition of $\{1, \ldots, 2 n\}$. Moreover we shall consider an expression more general than (4.1),

$$
\left\langle\Psi, A^{\varepsilon(1)}\left(\alpha_{1} \odot f_{1}\right) \cdots R(p) e^{i x \cdot p} \cdots A^{\varepsilon(2 n)}\left(\alpha_{2 n} \odot f_{2 n}\right) \Psi\right\rangle,
$$

where $R(p)$ is a polynomial. 
Since on the limit Hilbert module the left action is not defined, the most natural way to give a meaning to the quantity (4.6) is to define it as the limit of

$$
\begin{aligned}
&\left\langle\Phi, A_{\lambda}^{\varepsilon(1)}\left(S_{1}, T_{1}, f_{1}, a_{1}\right) \cdots A_{\lambda}^{\varepsilon(r)}\left(S_{r}, T_{r}, f_{r}, a_{r}\right) R(p) e^{i x \cdot p}\right. \\
&\left.A_{\lambda}^{\varepsilon(r+1)}\left(S_{r+1}, T_{r+1}, f_{r+1}, a_{r+1}\right) \cdots A_{\lambda}^{\varepsilon(2 n)}\left(S_{2 n}, T_{2 n}, f_{2 n}, a_{2 n}\right) \Phi\right\rangle .
\end{aligned}
$$

THEOREM 4.1. The limit of (4.6) exists and is equal to zero if $\varepsilon$ does not admit a noncrossing pair partition of $\{1, \ldots, 2 n\}$; while it is equal to

$$
\begin{gathered}
\prod_{h=1}^{n}\left\langle\chi_{\left[S_{m_{h}}, T_{m_{h}}\right]}, \chi_{\left[S_{m_{h}^{\prime}}, T_{m_{h}^{\prime}}\right)_{\mathbf{L}^{2}\left(\mathbb{R}_{+}\right)}} \int_{-\infty}^{+\infty} d u_{1} \ldots \int_{-\infty}^{+\infty} d u_{n} \int_{\mathbb{R}^{n d}} d k_{1} \ldots d k_{n} \prod_{h=1}^{n}\left(S_{u_{h}} f_{m_{h}}\right)\left(k_{h}\right) \bar{f}_{m_{h}^{\prime}}\left(k_{h}\right)\right. \\
\left\langle\xi, \prod_{h=1}^{n}\left[e^{i u_{h} k_{h} \cdot p} a_{2 h}\left(p+\sum_{l=1}^{n} k_{l} \chi_{\left(m_{l}^{\prime}, m_{l}\right)}(2 h)\right) a_{2 h+1}\left(p+\sum_{l=1}^{n} k_{l} \chi_{\left(m_{l}^{\prime}, m_{l}\right)}(2 h+1)\right)\right]\right. \\
\left.R\left(p+\sum_{l=1}^{n} k_{l} \chi_{\left[m_{l}^{\prime}, m_{l}\right]}(r)\right) e^{i x \cdot p} \eta\right\rangle \\
e^{i x \cdot \sum_{l=1}^{n} k_{l} \chi_{\left[m_{l}^{\prime}, m_{l}\right]}^{(r)}} \exp \left(i \sum_{h=1}^{n-1} \sum_{r=h+1}^{n} u_{h} k_{h} \cdot k_{r} \chi_{\left(m_{r}^{\prime}, m_{r}\right)}\left(m_{h}\right)\right)
\end{gathered}
$$

if $\varepsilon$ admits a (unique) noncrossing pair partition $\left\{m_{h}^{\prime}, m_{h}\right\}_{h=1}^{n}$.

Proof: The proof can be performed by putting together the argument from Section 2 and the technique used in [2].

\section{The stochastic differential equation}

By the same technique, we are able to prove our main result, i.e. the convergence of the matrix elements, in the collective number vectors, of the wave operator at time $t, U_{t / \lambda^{2}}^{(\lambda)}$, to a unitary operator $U(t)$ on the Fock module described in Sections 3 and 4 , satisfying the stochastic differential equation

$$
U(t)=1+\int_{0}^{t}\left(d A_{s}^{+}\left(\tilde{g}_{i p}\right)-d A_{s}\left(\tilde{g}_{i p}\right)-\left(\tilde{g}_{i p} \mid \tilde{g}_{i p}\right) \_j s\right) U(s)
$$

driven by the new quantum noise described in the previous section and where the quantum stochastic integrals are defined on the full $\mathcal{P}_{\Omega}$-Fock module described in Section 3 following the theory developed in [8-10]. As usual, in (5.1), the half-inner product $(\cdot \mid \cdot)_{-}$is defined replacing the $d t$-integral over $\mathbb{R}$, in (3.4), by an integral extended over the half line $(-\infty, 0]$, and for any polynomial $g_{p}=g(p)$ in $\mathcal{P}_{\Omega}, \tilde{g}_{i p}$ denotes the polynomial obtained from it by replacing $p$ by ip. 
In fact the argument in [3] guarantees that in order to consider the limit

$$
\left\langle\prod_{h=1}^{N} A_{\lambda}^{+}\left(S_{h}, T_{h}, f_{h}, a_{h}\right) \Phi, U_{t / \lambda^{2}}^{(\lambda)} \prod_{h=1}^{N^{\prime}} A_{\lambda}^{+}\left(S_{h}^{\prime}, T_{h}^{\prime}, f_{h}^{\prime}, a_{h}^{\prime}\right) \Phi\right\rangle
$$

one can replace the operator $U_{t / \lambda^{2}}^{(\lambda)}$ by its series expansion and take the limit (as $\lambda \rightarrow 0$ ) term by term, since any term has the form (4.6). Thus the techniques of [3] give the main result.

An interesting open problem is to determine the vacuum distribution of the classical random variable $A\left(\alpha \odot f_{a}\right)+A^{+}\left(\alpha \odot f_{a}\right)$ in the limit module.

\section{Note added on 14-01-2004}

The present paper was completed in April 1995 and endured various vicissitudes among which that of being lost. Now there is a revival of interest for the role of scattering and wave operators in the weak coupling limit of QED for applications to quantum optics and quantum information.

Therefore publication of these results, even after such a long delay, seems to be justified.

\section{REFERENCES}

[1] L. Accardi, Y. G. Lu and I. Volovich: Quantum Theory and its Stochastic Limit, Springer, Berlin 2002.

[2] L. Accardi and Y. G. Lu: From the weak coupling limit to a new type of quantum stochastic calculus, Quantum Probability and Related Topics, QP-PQ VII, 1-14, (1993), 1-14, Volterra preprint No. 68 (1991).

[3] L. Accardi and Y. G. Lu: The Wigner Semi-circle law in quantum electrodynamics, Commun. Math. Phys. 180 (1996), 605-632, Volterra preprint No 126 (1992).

[4] B. Blackadar: K-theory for Operator Algebras, Springer, Berlin 1986.

[5] J. Gough: The free stochastic limit of interacting quantum fields, Quantum Probability, Infinite Dimensional Analysis and Related Topics, Vol. 1, No. 3 (1998).

[6] J. Gough: The interacting-free quantum stochastic limit, J. Math. Phys. 38, 2 (1997), 867-881.

[7] T. Kato: Perturbation Theory for Linear Operators, Springer, New York-Berlin-Heidelberg 1966.

[8] Y. G. Lu: Quantum stochastic calculus on Hilbert module, Volterra preprint No 106 (1992).

[9] Y. G. Lu: The passage from quantum system with continuous spectrum to quantum Poisson processes on Hilbert module, J. Math. Phys. 36 (1) (1995), 142-176. Volterra preprint No 106 (1992).

[10] Y. G. Lu: A note on free stochastic calculus on Hilbert modules and its applications, Random Operators and Stoch. Eq., Vol. 1, No. 1, 1-8 (1996). Volterra Preprint 186, October 1994. 